\title{
FUNÇÃO DE AFILAMENTO E SORTIMENTOS DE MADEIRA PARA Araucaria angustifolia
}

\author{
TAPER FUNCTION AND TIMBER ASSORTMENTS FOR Araucaria angustifolia
}

\author{
Emanuel Arnoni Costa ${ }^{1}$ César Augusto Guimarães Finger ${ }^{2}$ Paulo Renato Schneider ${ }^{3}$ \\ André Felipe Hess ${ }^{4}$
}

\begin{abstract}
RESUMO
Funções de afilamento permitem determinar classes de dimensão e volumes das árvores com aplicação direta no planejamento das atividades técnicas e econômicas da floresta. Com esse propósito, os objetivos do presente estudo foram descrever o afilamento do fuste de Araucaria angustifolia (Bertol.) Kuntze na porção compreendida entre o nível do solo e a inserção da copa de árvores de remanescente florestal nativo, bem como ajustar modelo de regressão para a relação altura/DAP, para o diâmetro na inserção da copa sobre o DAP e demonstrar a aplicação das funções com a confecção de uma tabela de sortimentos de madeira. Oitenta e cinco árvores tiveram medidas seus diâmetros e alturas relativas pelo método de cubagem rigorosa de Hohenadl, assim como o diâmetro na inserção da copa com o Criterion RD 1000. Os modelos de afilamento ajustados por regressão foram o polinômio do $5^{\circ}$ grau, Kozak (1988), Lee et al. (2003) e Sharma e Zhang (2004). O coeficiente de determinação ajustado ( ${ }^{2}$ aj), o erro padrão da estimativa em porcentagem $(\mathrm{Syx} \%)$ e a análise gráfica entre valores estimados e observados foram as estatísticas usadas para avaliar o ajuste e a precisão dos modelos. O modelo da relação altura/DAP e do diâmetro no ponto de inserção da copa alcançaram boas estatísticas de ajuste e precisão, permitindo sua aplicação em inventários florestais e no planejamento da produção florestal. A flexibilidade e a eficiência do modelo de Kozak (1988) em predizer diâmetros e volumes até o ponto de inserção da copa favorecem o uso e obtenção de sortimentos de madeira de araucária.
\end{abstract}

Palavras-chave: pinheiro-brasileiro; perfil do fuste; volume individual; sortimentos de madeira.

\begin{abstract}
Taper functions allow determining size classes and volumes of trees with direct application in the planning of technical and economic forest activities. For this purpose, the aim of this study was to describe the taper of the trunk of Araucaria angustifolia (Bertol.) Kuntze in the portion between the ground level and the insertion of the crown of individuals in remnant of native forest. Also tried to fit the regression model to represent relations height/DBH, diameter at the insertion of the crown in function $\mathrm{DBH}$ and the development of assortment timber table. Eighty-five trees were measured their diameters and heights relatives using Hohenadl cubed method and the diameter of the insertion crown with Criterion RD 1000 instrument. The taper models were adjusted by regression analysis using polynomial of the $5^{\text {th }}$ degree, Kozak (1988), Lee et al. (2003) and Sharma and Zhang (2004). The adjusted coefficient of determination ( $\left.\mathrm{R}^{2} \mathrm{aj}\right)$, the
\end{abstract}

1 Engenheiro Florestal, Doutor do Programa de Pós-Graduação em Engenharia Florestal, Centro de Ciências Rurais, Universidade Federal de Santa Maria, Av. Roraima, 1000, CEP 97105-900, Santa Maria (RS), Brasil. emanuelarnonicost@hotmail.com

2 Engenheiro Florestal, Dr., Professor Titular do Departamento de Ciências Florestais, Centro de Ciências Rurais, Universidade Federal de Santa Maria, Av. Roraima, 1000, CEP 97105-900, Santa Maria (RS), Brasil. cesarfinger. ufsm@outlook.com

3 Engenheiro Florestal, Dr., Professor Titular do Departamento de Ciências Florestais, Centro de Ciências Rurais, Universidade Federal de Santa Maria, Av. Roraima, 1000, CEP 97105-900, Santa Maria (RS), Brasil. paulors@smail.ufsm.br

4 Engenheiro Florestal, Dr., Professor do Departamento de Engenharia Florestal, Centro de Ciências Agroveterinárias, Universidade do Estado de Santa Catarina, Av. Luiz de Camões, 2090, CEP 88520-000, Lages (SC), Brasil. a2afh@cav.udesc.br

Recebido para publicação em 8/02/2013 e aceito em 9/07/2014 
standard error of estimate in percentage (Syx\%) and graphical analysis between estimated values in function of observed were used statistics to evaluate the quality of fit and models precision. The model height/DBH and diameter at the insertion point of the crown evidence good fit statistical and precision, allowing its application in activities intended at planning and forest inventories. The flexibility and efficiency of Kozak (1988) model in predicting diameters and volumes until the crown insertion point favors the use and obtain timber assortments of araucaria.

Keywords: Parana pine; trunk profile; individual volume; timber assortments.

\section{INTRODUÇÃO}

Conhecer o afilamento do fuste das árvores é importante para o planejamento e execução de atividades florestais, principalmente quando se deseja classificar a produção pelas toras de madeira, segundo as metas estabelecidas para o talhão. Ao associá-lo à função de crescimento, a descrição matemática do afilamento permite inferir na quantidade e dimensão das toras de forma dinâmica, simulando cenários de custo e renda os quais podem auxiliar na definição da rotação da floresta.

$\mathrm{O}$ ajuste de modelos matemáticos capazes de descrever com acuidade a variação do afilamento das árvores tem ganhado importância na área florestal (BURKHART; TOMÉ, 2012). A partir da década de 1960, esses modelos têm sido aplicados com maior frequência; pois, com a integração da função, é possível estimar os diâmetros, ou diretamente o volume, em qualquer posição do fuste, conforme demonstraram Kozak, Munro e Smith (1969); Max e Burkhart (1976); Biging (1984); Kozak (1988); Lee et al. (2003) e Sharma e Zhang (2004).

Entretanto, as variações encontradas no afilamento do fuste das árvores decorrem de fatores regionais, climáticos, sítio, idade, tamanho da copa, posição social da copa, espécie e da densidade do povoamento (MUHAIRNE et al., 1994; KLOS et al., 2007; SHARMA; PARTON, 2009; NIGH; SMITH, 2012), sendo esta variabilidade um desafio na busca de acurácia na prognose dos sortimentos, principalmente quando se abordam árvores em florestas mistas e inequiâneas. O efeito da densidade da floresta sobre a forma geométrica foi corrigido por Sharma e Zhang (2004), com a inclusão de um coeficiente de regressão no modelo, que os levaram a obter estimativas mais acuradas que o de Kozak (1988). Kozak (1998), após uma década da publicação do modelo, incluiu um novo coeficiente de regressão que consistia de um di medido entre 40 a $50 \%$ da altura da árvore levando a melhorias nas estimativas dos diâmetros ao longo do fuste.
Diante do exposto, o presente estudo teve por objetivos descrever o afilamento do fuste de Araucaria angustifolia (Bertol.) Kuntze na porção compreendida entre o nível do solo e a inserção da copa de árvores de remanescente florestal nativo, bem como ajustar modelo de regressão para descrever a relação altura/DAP, para o diâmetro na inserção da copa sobre o DAP e demonstrar a aplicação das funções com a confecção de uma tabela de sortimentos de madeira.

\section{MATERIAL E MÉTODO}

\section{Área de estudo}

Os dados foram levantados em floresta nativa com 84 hectares localizada no município

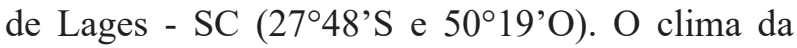
região é mesotérmico úmido (1.360 a $1.600 \mathrm{~mm})$, sem estação seca definida $(\mathrm{Cfb})$, com temperatura média anual entre 13,8 e $15,8^{\circ} \mathrm{C}$ e umidade relativa de $80 \%$ (EPAGRI, 2002). Os solos predominantes na região são nitossolos háplicos e cambissolos húmicos desenvolvidos a partir de rochas basálticas (EMBRAPA, 1999).

\section{Levantamento dos dados}

Oitenta e cinco árvores de araucária foram selecionadas no interior da floresta por um processo de amostragem inteiramente aleatório dentro de classes de diâmetro, recobrindo a amplitude de distribuição diamétrica previamente estabelecida por Hess et al. (2010), conforme representado na Figura 1. De cada árvore foram medidos: o diâmetro a altura do peito (DAP) com fita diamétrica; a altura (h); e, a altura de inserção da copa (hic), com o hipsômetro Vertex IV.

Para obtenção dos diâmetros superiores ao longo do fuste, considerou-se o método de cubagem rigorosa de Hohenadl com os diâmetros relativos (di) medidos a alturas específicas $\left(\mathrm{h}_{\mathrm{i}}\right)$ de $10 \%, 30 \%$, 

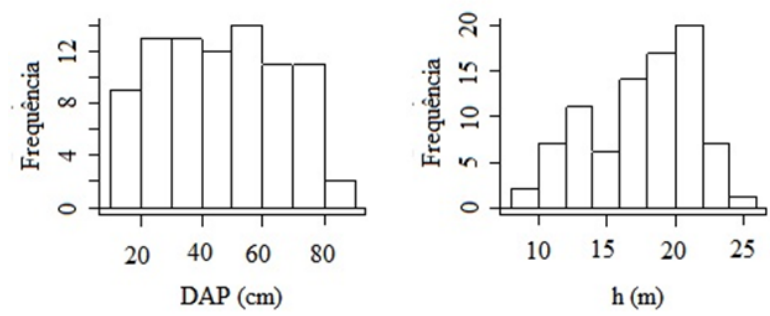

FIGURA 1: Frequência de árvores amostradas em classes de DAP e altura.

FIGURE 1: Frequency of trees sampled by DBH and height classes.

$50 \%, 70 \%$ da h, bem como o diâmetro na inserção da copa (dic). O diâmetro a $90 \%$ da altura não foi considerado por estar localizado dentro da copa. Nesta posição a presença de galhos dificultava e até mesmo impedia sua determinação dentro da floresta. As características das árvores avaliadas encontramse na Tabela 1.

A determinação dos valores $d_{i}$ e $h_{i}$ foi feita com o Criterion RD 1000 que possibilitou a cubagem das árvores em pé. Várias pesquisas utilizaram esse tipo de dendrômetro óptico em estudos de cubagem e de quantificação de biomassa de árvores (CLARK et al., 2000; KALLIOVIRTA et al., 2005). Este método ofereceu vantagens de não ser necessário abater a árvore e apresentar estimativas com pouca diferença entre o valor observado e o real (PARKEY; MATNEY, 1998; WILLIAMS et al., 1999).
TABELA 1: Características biométricas de 85 árvores avaliadas.

TABLE 1: Biometric characteristics of 85 trees evaluated.

\begin{tabular}{ccccc}
\hline Variáveis & Mínimo & Média & Máximo & CV\% \\
\hline DAP & 14,7 & 46,2 & 85,8 & 41,3 \\
h & 9,1 & 17,7 & 24,9 & 21,8 \\
dic & 9,6 & 29,5 & 61,0 & 43,4 \\
hic & 3,0 & 12,3 & 19,0 & 35,7 \\
\hline
\end{tabular}

Em que: DAP = diâmetro a altura do peito, $\mathrm{em} \mathrm{cm} ; \mathrm{h}=$ altura total, em m; dic = diâmetro na inserção da copa, em cm; hic = altura na inserção da copa, em m.

\section{Relação altura/DAP}

A relação altura/DAP foi descrita pelo modelo não linear de Michailoff (1) também empregado para araucária em floresta nativa por Costa et al. (2014) e o dic por modelo linear simples (2).

$$
\begin{gathered}
\mathrm{h}=1,3+\beta_{0} \exp \left(-\beta_{1} / \mathrm{DAP}\right)+\varepsilon \\
\operatorname{dic}=\beta_{0}+\beta_{1} \mathrm{DAP}+\varepsilon
\end{gathered}
$$

Em que: $\mathrm{h}=$ altura total, em $\mathrm{m}$; dic = diâmetro na inserção da copa, em cm; DAP = diâmetro a altura do peito, em $\mathrm{cm} ; \beta_{0}$ e $\beta_{1}=$ parâmetros a serem estimados; $\varepsilon=$ erro.

TABELA 2: Modelos de afilamento para araucária.

TABLE 2: Taper models for araucaria.



Em que: $\mathrm{h}=$ altura total; $\mathrm{hi}=$ altura relativa; $\mathrm{DAP}=$ diâmetro a altura do peito; di $=$ diâmetro relativo; $\beta_{0 .} . \beta_{7}=$ são coeficientes estimados; $\varepsilon=$ erro; $\ln =\operatorname{logaritmo}$ natural; $p=$ ponto de inflexão considerado em $1,3 / \mathrm{h}$. 


\section{Modelos de afilamento}

Os modelos ajustados para avaliar o afilamento do fuste de araucária (Tabela 2) incluíram modelos polinomial (linear) e não linear.

$\mathrm{O}$ volume total da árvore até o ponto de inserção da copa (vtic) foi determinado pela integral quando usado o polinômio de $5^{\circ}$ grau:

$$
\text { vtic }=\mathrm{K} \int_{\mathrm{h}_{1}}^{\mathrm{h}_{2}} \mathrm{~d}^{2} \delta \mathrm{h}
$$

vtic $=\mathrm{K} \mathrm{DAP} 2 \int_{\mathrm{h}_{1}}^{\mathrm{h}_{2}}\left(\mathrm{c}_{\mathrm{o}}+\mathrm{c}_{1} \mathrm{~h}_{1}^{\mathrm{p}_{1}}+\mathrm{c}_{2} \mathrm{~h}_{2}^{\mathrm{p}_{2}}+\ldots+\mathrm{c}_{\mathrm{n}} \mathrm{h}_{\mathrm{n}}^{\mathrm{p}_{\mathrm{n}}}\right)^{2} \delta \mathrm{h}$

Em que: $\mathrm{K}=\pi / 40000 ; \mathrm{p}_{\mathrm{i}}=$ expoentes variando de 1 a $5 ; c_{o}=\beta_{0} ; c_{1}=\beta_{1} / h^{1} ; c_{2}=\beta_{2} / h^{2} ; \ldots ; c_{5}=\beta_{5} / h^{5}$.

Resolvendo a integral, tem-se:

$$
\text { vtic }=\text { K.DAP }\left[\begin{array}{l}
c_{0}^{2} h_{\mathrm{i}}+c_{0} c_{1} \mathrm{~h}_{\mathrm{i}}^{2}+\left(\frac{2}{3} c_{0} c_{2}+\frac{1}{3} c_{1}^{2}\right) \mathrm{h}_{\mathrm{i}}^{3}+\left(\frac{1}{2} c_{0} c_{3}+\frac{1}{2} c_{1} c_{2}\right) \mathrm{h}_{\mathrm{i}}^{4}+ \\
\left(\frac{2}{5} c_{0} c_{4}+\frac{2}{5} c_{1} c_{3}+\frac{1}{5} c_{2}^{2}\right) h_{\mathrm{i}}^{5}+\left(\frac{1}{3} c_{0} c_{5}+\frac{1}{3} c_{1} c_{4}+\frac{1}{3} c_{2} c_{3}\right)_{i}^{6}+ \\
\left(\frac{2}{7} c_{1} c_{5}+\frac{2}{7} c_{2} c_{4}+\frac{1}{7} c_{3}^{2}\right) h_{i}^{7}+\left(\frac{1}{4} c_{2} c_{5}+\frac{1}{4} c_{3} c_{4}\right) h_{i}^{8}+ \\
\left(\frac{2}{9} c_{3} c_{5}+\frac{1}{9} c_{4}^{2}\right) h_{i}^{9}+\left(\frac{1}{5} c_{4} c_{5}\right) h_{i}^{10}+\left(\frac{1}{11} c_{5}^{2}\right) h_{i}^{11}
\end{array}\right]_{h_{1}}^{h_{2}}
$$

Os modelos de afilamento 4,5 , e 6 não são analiticamente integráveis necessitando empregar técnicas de integração numérica para obter os volumes parciais (vi) e totais (vtic) (THOMAS; WIER; HASS, 2010). Neste trabalho, para facilitar o cálculo do vtic de cada árvore, dividiu-se o fuste em cem seções e a fórmula de Smalian foi usada para calcular o volume de cada seção (vi). A soma dessas seções resultou no vtic da árvore conforme empregado por Li e Weiskittel (2010) e Li et al. (2012).

\section{Escolha do melhor modelo ajustado}

As estatísticas foram calculadas no SAS V. 9.1 (SAS INSTITUTE, 2004), sendo considerados para avaliar o ajuste e a precisão dos modelos testados, o coeficiente de determinação ajustado ( $\mathrm{R}^{2} \mathrm{aj}$.), o erro padrão da estimativa em porcentagem (Syx\%) e a análise gráfica entre valores estimados em função dos observados. Essa análise é de ampla importância, pois valores menos dispersos em relação à linha de referência com intercepto zero e inclinação de $45^{\circ}$ são preferíveis.

Para a variável dependente que sofreu algum tipo de transformação fez-se o recálculo do $\mathrm{R}^{2}$ aj. e Syx\% no programa Microsoft Office Excel calculando os resíduos diretamente com os valores observados e estimados para que essas estatísticas possam ser comparadas com as equações cuja variável dependente não sofreu transformação (Tabela 3).

\section{Sortimentos de madeira}

Embora os sortimentos possam ser redefinidos para qualquer dimensão, após a seleção dos modelos de afilamento, a título de exemplo, foram considerados os sortimentos com casca: $\mathrm{S} 1$ = tora para serraria com diâmetro na ponta fina maior e igual a $40 \mathrm{~cm}$ e comprimento de 5,4 metros; $\mathrm{S} 2$ = tora para serraria com diâmetro na ponta fina maior ou igual que $30 \mathrm{~cm}$ e menor que $40 \mathrm{~cm}$ e comprimento de 2,7 metros; $\mathrm{S} 3$ = tora com diâmetro na ponta fina maior ou igual a $20 \mathrm{~cm}$ e menor que

TABELA 3: Estatísticas de ajuste e precisão dos modelos de afilamento.

TABLE 3: Statistics of fit and precision taper models.

\begin{tabular}{ccc}
\hline Estatísticas & Fórmula & Ideal \\
\hline$R^{2}$ aj. & $1-\left[\left(\frac{\sum_{i=1}^{\mathrm{n}}\left(\mathrm{y}_{\mathrm{i}}-\hat{\mathrm{y}}\right)^{2}}{\sum_{\mathrm{i}=1}^{\mathrm{n}}\left(\mathrm{y}_{\mathrm{i}}-\overline{\mathrm{y}}\right)^{2}}\right) \cdot\left(\frac{\mathrm{n}-1}{\mathrm{n}-\mathrm{p}}\right)\right]$ & 1 \\
\hline Syx $\%$ & $\left(\sqrt{\frac{\sum_{\mathrm{i}=1}^{\mathrm{n}}\left(\mathrm{y}_{\mathrm{i}}-\hat{\mathrm{y}}\right)^{2}}{\mathrm{n}-\mathrm{p}} / \mathrm{y}} / .100\right.$ & 0 \\
\hline
\end{tabular}

Em que: $\mathrm{y}_{\mathrm{i}}=$ di e vtic observado; $\hat{\mathrm{y}}=$ di e vtic estimado; $\overline{\mathrm{y}}=$ di e vtic médio observado; $\mathrm{n}=$ número de observações; $\mathrm{p}=$ número de coeficientes do modelo. 
$30 \mathrm{~cm}$ e comprimento de 2,2 metros; $\mathrm{S} 4$ = tora para energia ou indústria com diâmetro na ponta fina menor que $20 \mathrm{~cm}$ e com restante do comprimento disponível do fuste. No estabelecimento dos sortimentos, foi priorizada a formação de toras com maior diâmetro e comprimento, na sequência $\mathrm{S} 1$, $\mathrm{S} 2, \mathrm{~S} 3$ e S4.

\section{RESULTADOS E DISCUSSÕES}

\section{Estimativa da altura da árvore e do diâmetro na inserção da copa}

A relação altura/DAP, fundamental para o desenvolvimento de modelos de crescimento e produção florestal além de sua aplicação em inventários florestais, teve os parâmetros estimados significantes a 5\% com $\mathrm{R}^{2}$ aj. de 0,562 e Syx $\%$ de $14,4 \%$ (Tabela 4). Esses valores foram similares aos encontrados por Machado et al. (2008) em um fragmento de Floresta Ombrófila Mista, no estado do Paraná.

A maior dispersão das alturas estimadas (Figura 2) versus as observadas reflete a condição das árvores terem sido amostradas em diferentes sítios, idades e intensidades de competição no interior da floresta. Essa grande variabilidade foi citada por Heinsdijk (1959) que testou esta relação em araucárias do estrato dominante e dominado, que visualmente mostravam tendências desiguais. Costa et al. (2014) determinaram estatisticamente que árvores dominantes, codominantes e dominadas possuíam funções com níveis e inclinações distintos, tendo recomendando ajustes de modelos altura/DAP separados para obter maior acurácia nas estimativas.

As estatísticas de ajuste e precisão do modelo 2 que descreve o dic em função do DAP, alcançou valor de $\mathrm{R}^{2}$ aj. de 0,899 e Syx\% de 13,8\%, mas o modelo teve apenas o coeficiente angular significante a $5 \%$ de probabilidade (Tabela 4). A relação linear crescente descrita pelo modelo 2 mostrou que o aumento de um centímetro no DAP resulta no aumento de $0,68 \mathrm{~cm}$ no dic.
A análise gráfica entre os valores do dic estimados versus os observados mostrou distribuição regular, sem tendenciosidade, para a amplitude diamétrica avaliada (Figura 2).
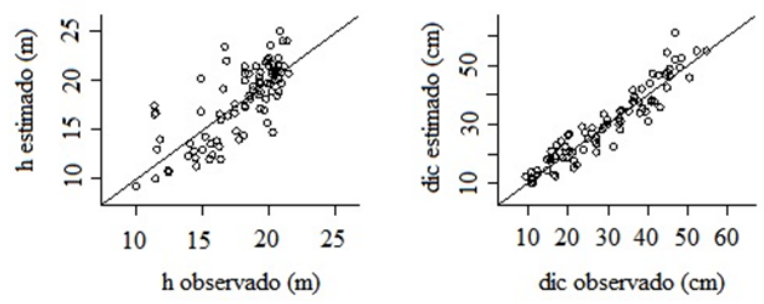

FIGURA 2: Altura (h) e diâmetro na inserção da copa (dic) estimado pelas equações 1 e 2 em função dos valores observados.

FIGURE 2: Height (h) and diameter at crown insertion (dic) estimated by equations 1 and 2 in fuction of the observed values.

\section{Estimativa de diâmetros relativos (di) e de volume até a inserção da copa (vtic)}

Os quatro modelos ajustados mostraram pequena variação nos valores de $\mathrm{R}^{2} \mathrm{aj} . \approx 98 \%$ com Syx $\%$ entre 6,8 a $7,5 \%$. Apesar de a regularidade das estimativas apresentadas pelos modelos, foram selecionados o 4 e o 6 , referentes ao de Kozak e Sharma e Zhang. Esses apresentaram, respectivamente, os coeficientes $\beta_{3}$ e $\beta_{2}$ não significantes $\alpha=5 \%$, sendo então eliminados da estrutura original e, a seguir, os modelos foram recalculados (Tabela 5).

As estatísticas $\mathrm{R}^{2} a j$. e Syx\% analisadas separadamente não permitiram definir claramente a equação de afilamento ideal, tendo sido necessário avaliar sua capacidade de predizer di e o vtic. Assim, a predição dos di sem influência da transformação da variável dependente foi mostrada sobre valores observados (Figura 3), na qual se verificou semelhança na distribuição dos valores entre os modelos.

As estatísticas de $\mathrm{R}^{2} \mathrm{aj}$. e Syx\% obtidas do vtic (Tabela 6) calculado pelo método de Smalian

TABELA 4: Coeficientes dos modelos 1 e 2 e estatísticas de ajuste e precisão.

TABLE 4: Coefficients of models 1 and 2,statistics fit and precision.

\begin{tabular}{cccccc}
\hline Modelo & Variável & $\beta_{0}$ & $\beta_{1}$ & $\mathrm{R}^{2}$ aj. & Syx $\%$ \\
\hline 1 & $\mathrm{H}$ & 23,9174 & 14,8902 & 0,562 & 14,4 \\
2 & Dic & $0,0412^{\text {ns }}$ & 0,6376 & 0,899 & 13,8 \\
\hline
\end{tabular}

Em que: $\mathrm{h}=$ altura total, em $\mathrm{m}$; dic = diâmetro do fuste no ponto de inserção de copa, em $\mathrm{cm}$; ns = coeficiente não significante a $\mathrm{P} \leq 0,05$. 
TABELA 5: Coeficientes ajustados, estatísticas de ajuste e precisão dos modelos de afilamento.

TABLE 5: Adjusted coefficients, statistics fit and precision of the taper models.

\begin{tabular}{ccccccccccc}
\hline Eq. & $\beta_{0}$ & $\beta_{1}$ & $\beta_{2}$ & $\beta_{3}$ & $\beta_{4}$ & $\beta_{5}$ & $\beta_{6}$ & $\beta_{7}$ & $\mathrm{R}^{2}$ aj. & Syx $\%$ \\
\hline 3 & 1,1835 & $-3,2691$ & 14,6593 & $-34,9980$ & 37,8678 & $-15,1253$ & - & - & 0,975 & 7,5 \\
4 & 0,8944 & 1,0361 & 0,9994 & ns & $-0,1885$ & 1,1267 & $-0,2960$ & $-0,0420$ & 0,980 & 6,8 \\
5 & - & 1,1146 & 1,0016 & 1,2270 & $-2,2292$ & 1,4031 & - & - & 0,974 & 7,7 \\
6 & 1,0005 & 2,0946 & ns & $-0,4033$ & - & - & - & - & 0,976 & 7,4 \\
\hline
\end{tabular}

Em que: Eq.= equação; $n s=$ coeficientes não significante a $\mathrm{P} \leq 0,05$.

TABELA 6: Valor das estatísticas de ajuste e precisão dos modelos de afilamento.

TABLE 6: Statistics fit value and precision taper models.

\begin{tabular}{cccc}
\hline Modelo & Variável & $\mathrm{R}^{2}$ aj. & Syx\% \\
\hline 3 & & 0,975 & 19,2 \\
4 & \multirow{2}{*}{ vtic } & 0,979 & 17,4 \\
5 & & 0,976 & 18,7 \\
6 & & 0,968 & 21,5 \\
\hline
\end{tabular}

Em que: vtic $=$ volume total até o ponto de inserção da copa, em m³.cc.


FIGURA 3: Valores dos di estimados em função dos observados para os modelos: polinômio do $5^{\circ}$, Kozak, Lee et al. e Sharma e Zhang.

FIGURE 3: Estimated values of di in function observed for models: polynomial of $5^{\circ}$, Kozak, Lee et al. and Sharma and Zhang.

com a soma do volume de cada seção mostrou melhores ajustes com as equações 4 e 5 , modelos de Kozak e Lee et al., respectivamente, com $\mathrm{R}^{2}$ aj. na ordem de $\approx 98 \%$ e Syx $\%$ entre 17,4 a $18,7 \%$.

A análise gráfica dos vtic estimados sobre os observados confirmou a superioridade de Kozak com valores de menor dispersão em relação à linha de referência quando comparada com o polinômio de $5^{\circ}$ grau, Lee et al. e Sharma e Zhang (Figura 4).

O resultado mostrou a importância de avaliar a acurácia das estimativas dos diâmetros (di) e dos volumes (vtic) na seleção do modelo de afilamento, ainda que os modelos apresentassem estimativas de di similares (com pequena variação entre modelos) quando confrontados com valores de vi, ficou evidenciado a superioridade do modelo de Kozak em relação aos demais.

\section{Seleção do modelo de afilamento}

Entre os modelos testados o de Kozak (Eq.4) apresentou maior precisão de estimativas para os dados deste estudo. O polinômio de $5^{\circ}$ grau, normalmente empregado, para descrever o afilamento de espécies florestais no Brasil devido à precisão e facilidade de aplicação, (FIGUEIREDOFILHO, 1996; SCHNEIDER et al., 1996; MÜLLER, 2004; MIGUEL et al., 2011; SOUZA et al., 2012) apresentou resultados inferiores ao de Kozak.

$\mathrm{Na}$ estimativa dos di, o polinômio de $5^{\circ}$ grau e o de Lee et al. apresentaram valores com maior dispersão em toda a amplitude das classes de diâmetro (Figura 3). Apesar de não ser o melhor para descrever o afilamento de araucária, o modelo de Lee et al. (2003) tem coeficientes com fundamentação analítica, permitindo descrever diferentes formas geométricas, variando da base para o ápice, desde 

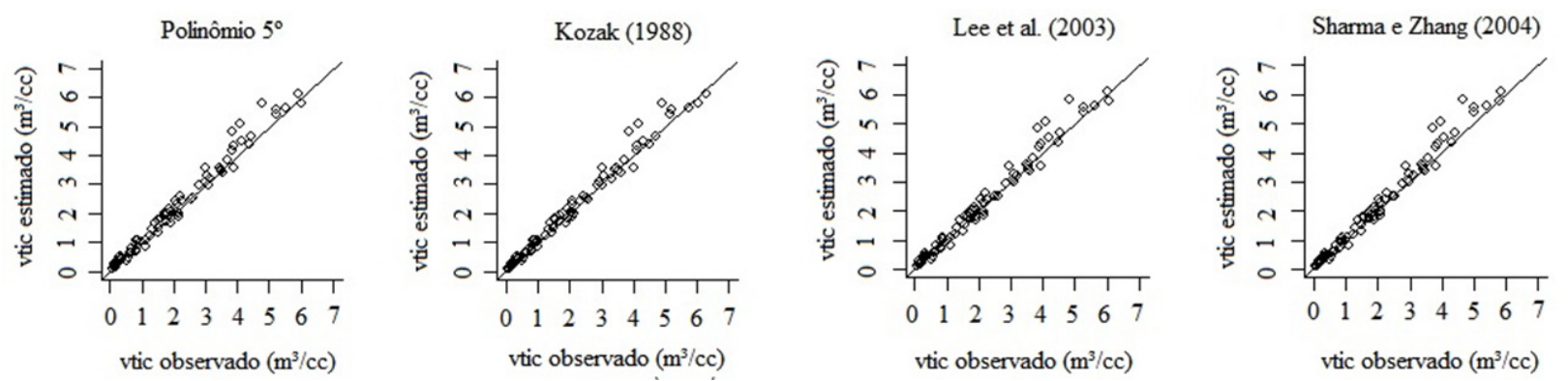

FIGURA 4: Valores do vtic estimado em função dos observados para os modelos: polinômio do $5^{\circ}$, Kozak, Lee et al. e Sharma e Zhang.

FIGURE 4: Estimated values of vtic in function observed for models: polynomial of $5^{\circ}$, Kozak, Lee et al. and Sharma and Zhang.

o neiloide, um paraboloide na parte intermediária, passando a um cone na parte superior.

$\mathrm{O}$ efeito da densidade da floresta sobre a forma geométrica de Jack pine, Balsam Fir e Black spruce foi avaliado por Sharma e Zhang (2004) com a inclusão de um coeficiente de regressão no modelo, que levou a obter estimativas mais acuradas que o de Kozak (1988). No entanto, no presente estudo em araucária, o modelo de Kozak (Eq.4) mostrou claramente o melhor ajuste com menores desvios. A flexibilidade e a boa performance desse modelo principalmente para estimativas na parte inferior do fuste favorecem sua aplicação a árvores de grandes dimensões que apresentam maior volume de madeira nesta porção.

\section{Sortimentos de madeira}

Para explicar a elevada variação da relação altura/DAP decorrente de fatores do ambiente, idade, competição, área basal e outros; duas curvas proporcionais à linha média de regressão (Eq.1) foram geradas: uma acima e outra abaixo, descrevendo a tendência máxima, média e mínima das alturas amostradas. Esse procedimento, comum na formação de tabelas de sortimento, como encontrado em Schneider (1984), Müller (2004), Souza (2009) e Schneider (2012), permitiu reduzir os erros na formação do sortimento de madeira (Tabela 7).

A classificação dos sortimentos dos fustes

TABELA 7: Sortimentos de madeira do fuste para araucária.

TABLE 7: Assortments of wood bole for araucaria.

\begin{tabular}{|c|c|c|c|c|c|c|c|c|c|c|c|}
\hline $\mathrm{d}$ & $\mathrm{h}$ & hic & \multirow{2}{*}{$\mathrm{n}$} & \multicolumn{2}{|c|}{ VS1 } & VS2 & \multirow{2}{*}{$\mathrm{n}$} & \multirow{2}{*}{$\begin{array}{c}\text { VS3 } \\
\% \\
\end{array}$} & \multicolumn{2}{|c|}{ VS4 } & \multirow{2}{*}{$\begin{array}{c}\text { vtic } \\
\left(\mathrm{m}^{3} . \mathrm{cc}\right)\end{array}$} \\
\hline$(\mathrm{cm})$ & $(\mathrm{m})$ & $(\mathrm{m})$ & & $\%$ & $\mathrm{n}$ & $\%$ & & & $\left(\mathrm{~m}^{3} . \mathrm{cc}\right)$ & $\%$ & \\
\hline \multirow{3}{*}{20,0} & 10,4 & 6,7 & 0 & 0,0 & 0 & 0,0 & 0 & 0,0 & 0,1510 & 100,0 & 0,1510 \\
\hline & 12,4 & 7,7 & 0 & 0,0 & 0 & 0,0 & 0 & 0,0 & 0,1695 & 100,0 & 0,1695 \\
\hline & 14,4 & 8,7 & 0 & 0,0 & 0 & 0,0 & 0 & 0,0 & 0,1879 & 100,0 & 0,1879 \\
\hline \multirow{3}{*}{22,0} & 11,2 & 7,3 & 0 & 0,0 & 0 & 0,0 & 1 & 42,4 & 0,1136 & 57,6 & 0,1973 \\
\hline & 13,2 & 8,3 & 0 & 0,0 & 0 & 0,0 & 1 & 38,0 & 0,1361 & 62,0 & 0,2197 \\
\hline & 15,2 & 9,3 & 0 & 0,0 & 0 & 0,0 & 1 & 34,5 & 0,1586 & 65,5 & 0,2421 \\
\hline \multirow{3}{*}{24,0} & 11,9 & 7,8 & 0 & 0,0 & 0 & 0,0 & 1 & 40,0 & 0,1505 & 60,0 & 0,2508 \\
\hline & 13,9 & 8,8 & 0 & 0,0 & 0 & 0,0 & 2 & 63,3 & 0,1019 & 36,7 & 0,2774 \\
\hline & 15,9 & 9,8 & 0 & 0,0 & 0 & 0,0 & 2 & 58,2 & 0,1272 & 41,8 & 0,3041 \\
\hline \multirow{3}{*}{26,0} & 12,5 & 8,3 & 0 & 0,0 & 0 & 0,0 & 2 & 66,2 & 0,1055 & 33,8 & 0,3119 \\
\hline & 14,5 & 9,3 & 0 & 0,0 & 0 & 0,0 & 2 & 60,6 & 0,1351 & 39,4 & 0,3434 \\
\hline & 16,5 & 10,3 & 0 & 0,0 & 0 & 0,0 & 3 & 75,8 & 0,0908 & 24,2 & 0,3748 \\
\hline \multirow{3}{*}{28,0} & 13,1 & 7,8 & 0 & 0,0 & 0 & 0,0 & 3 & 90,8 & 0,0326 & 9,2 & 0,3551 \\
\hline & 15,1 & 9,8 & 0 & 0,0 & 0 & 0,0 & 3 & 78,6 & 0,0893 & 21,4 & 0,4178 \\
\hline & 17,1 & 11,8 & 0 & 0,0 & 0 & 0,0 & 4 & 84,9 & 0,0722 & 15,1 & 0,4768 \\
\hline \multirow{3}{*}{30,0} & 13,6 & 8,2 & 0 & 0,0 & 0 & 0,0 & 3 & 87,5 & 0,0536 & 12,5 & 0,4291 \\
\hline & 15,6 & 10,2 & 0 & 0,0 & 0 & 0,0 & 4 & 92,1 & 0,0398 & 7,9 & 0,5008 \\
\hline & 17,6 & 12,2 & 0 & 0,0 & 0 & 0,0 & 4 & 82,8 & 0,0977 & 17,2 & 0,5687 \\
\hline
\end{tabular}

Continua... 
TABELA 7: Continuação...

TABLE 7: Continued...

\begin{tabular}{|c|c|c|c|c|c|c|c|c|c|c|c|}
\hline \multirow{2}{*}{$\frac{\mathrm{d}}{(\mathrm{cm})}$} & \multirow{2}{*}{$\frac{\mathrm{h}}{(\mathrm{m})}$} & \multirow{2}{*}{$\begin{array}{l}\text { hic } \\
\text { (m) }\end{array}$} & \multirow{2}{*}{$\mathrm{n}$} & \multicolumn{2}{|l|}{ VS1 } & VS2 & \multirow{2}{*}{$\mathrm{n}$} & \multirow{2}{*}{$\begin{array}{c}\text { VS3 } \\
\%\end{array}$} & \multicolumn{2}{|c|}{ VS4 } & \multirow{2}{*}{$\begin{array}{c}\text { vtic } \\
\left(\mathrm{m}^{3} \cdot \mathrm{cc}\right.\end{array}$} \\
\hline & & & & $\%$ & $\mathrm{n}$ & $\%$ & & & $\left(\mathrm{~m}^{3} . \mathrm{cc}\right)$ & $\%$ & \\
\hline \multirow{3}{*}{32,0} & 14,1 & 8,7 & 0 & 0,0 & 0 & 0,0 & 4 & 100,0 & 0,0000 & 0,0 & 0,5194 \\
\hline & 16,1 & 10,7 & 0 & 0,0 & 1 & 36,5 & 3 & 56,3 & 0,0424 & 7,1 & 0,5933 \\
\hline & 18,1 & 12,7 & 0 & 0,0 & 1 & 32,3 & 4 & 63,0 & 0,0315 & 4,7 & 0,6705 \\
\hline \multirow{3}{*}{34,0} & 14,6 & 9,1 & 0 & 0,0 & 1 & 40,8 & 2 & 45,4 & 0,0832 & 13,8 & 0,6024 \\
\hline & 16,6 & 11,1 & 0 & 0,0 & 1 & 35,4 & 3 & 55,4 & 0,0642 & 9,2 & 0,6941 \\
\hline & 18,6 & 13,1 & 0 & 0,0 & 1 & 31,4 & 4 & 62,2 & 0,0499 & 6,4 & 0,7813 \\
\hline \multirow{3}{*}{36,0} & 15,0 & 9,5 & 0 & 0,0 & 1 & 39,4 & 3 & 60,3 & 0,0025 & 0,4 & 0,7014 \\
\hline & 17,0 & 11,5 & 0 & 0,0 & 2 & 60,6 & 2 & 31,2 & 0,0662 & 8,2 & 0,8040 \\
\hline & 19,0 & 13,5 & 0 & 0,0 & 2 & 54,4 & 3 & 39,9 & 0,0512 & 5,7 & 0,9017 \\
\hline \multirow{3}{*}{38,0} & 15,4 & 9,8 & 0 & 0,0 & 2 & 67,0 & 1 & 18,6 & 0,1167 & 14,4 & 0,8089 \\
\hline & 17,4 & 11,8 & 0 & 0,0 & 2 & 59,1 & 2 & 30,9 & 0,0922 & 10,0 & 0,9231 \\
\hline & 19,4 & 13,8 & 0 & 0,0 & 2 & 53,2 & 3 & 39,7 & 0,0734 & 7,1 & 1,0319 \\
\hline & 15,8 & 10,2 & 0 & 0,0 & 2 & 65,2 & 2 & 33,2 & 0,0143 & 1,5 & 0,9251 \\
\hline 40,0 & 17,8 & 12,2 & 0 & 0,0 & 3 & 78,0 & 1 & 13,2 & 0,0927 & 8,8 & 1,0513 \\
\hline & 19,8 & 14,2 & 0 & 0,0 & 3 & 70,8 & 2 & 22,9 & 0,0733 & 6,3 & 1,1719 \\
\hline & 16,1 & 10,5 & 0 & 0,0 & 3 & 85,4 & 1 & 14,1 & 0,0050 & 0,5 & 1,0500 \\
\hline 42,0 & 18,1 & 12,5 & 0 & 0,0 & 3 & 76,5 & 1 & 13,2 & 0,1226 & 10,3 & 1,1888 \\
\hline & 20,1 & 14,5 & 0 & 0,0 & 3 & 69,7 & 2 & 22,8 & 0,0990 & 7,5 & 1,3216 \\
\hline & 16,4 & 10,8 & 0 & 0,0 & 3 & 83,8 & 1 & 14,0 & 0,0261 & 2,2 & 1,1835 \\
\hline 44,0 & 18,4 & 12,8 & 0 & 0,0 & 3 & 75,2 & 2 & 23,9 & 0,0113 & 0,8 & 1,3357 \\
\hline & 20,4 & 14,8 & 0 & 0,0 & 4 & 83,5 & 1 & 10,0 & 0,0967 & 6,5 & 1,4813 \\
\hline & 16,7 & 11,1 & 0 & 0,0 & 3 & 82,2 & 1 & 14,0 & 0,0502 & 3,8 & 1,3259 \\
\hline 46,0 & 18,7 & 13,1 & 1 & 54,7 & 2 & 35,0 & 0 & 0,0 & 0,1539 & 10,3 & 1,4998 \\
\hline & 20,7 & 15,1 & 1 & 49,7 & 3 & 44,7 & 0 & 0,0 & 0,0919 & 5,5 & 1,6585 \\
\hline & 17,0 & 11,4 & 1 & 60,0 & 2 & 37,6 & 0 & 0,0 & 0,0362 & 2,4 & 1,4859 \\
\hline 48,0 & 19,0 & 13,4 & 1 & 53,8 & 2 & 34,8 & 1 & 10,4 & 0,0181 & 1,1 & 1,6659 \\
\hline & 21,0 & 15,4 & 1 & 48,9 & 3 & 44,5 & 0 & 0,0 & 0,1201 & 6,5 & 1,8385 \\
\hline & 17,2 & 11,6 & 1 & 58,9 & 2 & 37,3 & 0 & 0,0 & 0,0623 & 3,8 & 1,6466 \\
\hline 50,0 & 19,2 & 13,6 & 1 & 52,9 & 3 & 47,0 & 0 & 0,0 & 0,0012 & 0,1 & 1,8415 \\
\hline & 21,2 & 15,6 & 1 & 48,2 & 3 & 44,3 & 0 & 0,0 & 0,1513 & 7,5 & 2,0285 \\
\hline & 17,4 & 11,9 & 1 & 57,9 & 2 & 37,1 & 0 & 0,0 & 0,0916 & 5,0 & 1,8161 \\
\hline 52,0 & 19,4 & 13,9 & 1 & 52,1 & 3 & 46,8 & 0 & 0,0 & 0,0218 & 1,1 & 2,0265 \\
\hline & 21,4 & 15,9 & 1 & 47,6 & 3 & 44,1 & 1 & 8,1 & 0,0046 & 0,2 & 2,2285 \\
\hline & 17,6 & 12,1 & 1 & 57,0 & 2 & 36,8 & 0 & 0,0 & 0,1239 & 6,2 & 1,9945 \\
\hline 54,0 & 19,6 & 14,1 & 1 & 51,4 & 3 & 46,6 & 0 & 0,0 & 0,0449 & 2,0 & 2,2209 \\
\hline & 21,6 & 16,1 & 1 & 46,9 & 3 & 43,9 & 1 & 8,2 & 0,0242 & 1,0 & 2,4384 \\
\hline & 17,8 & 12,3 & 1 & 56,1 & 2 & 36,6 & 0 & 0,0 & 0,1593 & 7,3 & 2,1818 \\
\hline 56,0 & 19,8 & 14,3 & 1 & 50,7 & 3 & 46,4 & 0 & 0,0 & 0,0706 & 2,9 & 2,4247 \\
\hline & 21,8 & 16,3 & 2 & 78,1 & 2 & 21,9 & 0 & 0,0 & 0,0013 & 0,0 & 2,6585 \\
\hline & 18,0 & 12,5 & 1 & 55,3 & 2 & 36,4 & 0 & 0,0 & 0,1979 & 8,3 & 2,3780 \\
\hline 58,0 & 20,0 & 14,5 & 2 & 83,7 & 1 & 12,5 & 0 & 0,0 & 0,0989 & 3,7 & 2,6382 \\
\hline & 22,0 & 16,5 & 2 & 77,4 & 2 & 21,9 & 0 & 0,0 & 0,0213 & 0,7 & 2,8883 \\
\hline & 18,1 & 12,7 & 1 & 54,5 & 2 & 36,2 & 0 & 0,0 & 0,2397 & 9,3 & 2,5831 \\
\hline 60,0 & 20,1 & 14,7 & 2 & 82,9 & 1 & 12,6 & 0 & 0,0 & 0,1298 & 4,5 & 2,8610 \\
\hline & 22,1 & 16,7 & 2 & 76,7 & 2 & 21,9 & 0 & 0,0 & 0,0434 & 1,4 & 3,1280 \\
\hline & 18,3 & 12,9 & 2 & 89,8 & 0 & 0,0 & 0 & 0,0 & 0,2847 & 10,2 & 2,7975 \\
\hline 62,0 & 20,3 & 14,9 & 2 & 82,2 & 1 & 12,6 & 0 & 0,0 & 0,1633 & 5,3 & 3,0931 \\
\hline & 22,3 & 16,9 & 2 & 76,0 & 2 & 22,0 & 0 & 0,0 & 0,0677 & 2,0 & 3,3777 \\
\hline & 18,4 & 13,1 & 2 & 89,0 & 0 & 0,0 & 0 & 0,0 & 0,3330 & 11,0 & 3,0204 \\
\hline 64,0 & 20,4 & 15,1 & 2 & 81,4 & 1 & 12,6 & 0 & 0,0 & 0,1995 & 6,0 & 3,3347 \\
\hline & 22,4 & 17,1 & 2 & 75,4 & 2 & 22,0 & 0 & 0,0 & 0,0941 & 2,6 & 3,6373 \\
\hline & 18,5 & 13,3 & 2 & 88,2 & 0 & 0,0 & 1 & 11,1 & 0,0229 & 0,7 & 3,2524 \\
\hline 66,0 & 20,5 & 15,3 & 2 & 80,8 & 1 & 12,6 & 0 & 0,0 & 0,2384 & 6,6 & 3,5857 \\
\hline & 22,5 & 17,3 & 2 & 74,8 & 2 & 22,0 & 0 & 0,0 & 0,1227 & 3,1 & 3,9068 \\
\hline & 18,6 & 13,4 & 2 & 87,4 & 0 & 0,0 & 1 & 11,1 & 0,0503 & 1,4 & 3,4932 \\
\hline 68,0 & 20,6 & 15,4 & 2 & 80,1 & 1 & 12,6 & 0 & 0,0 & 0,2799 & 7,3 & 3,8462 \\
\hline & 22,6 & 17,4 & 2 & 74,3 & 2 & 22,1 & 0 & 0,0 & 0,1535 & 3,7 & 4,1863 \\
\hline
\end{tabular}

Continua... 
TABELA 7: Continuação...

TABLE 7: Continued...

\begin{tabular}{|c|c|c|c|c|c|c|c|c|c|c|c|}
\hline $\mathrm{d}$ & $\mathrm{h}$ & hic & \multirow{2}{*}{$\mathrm{n}$} & \multicolumn{2}{|l|}{ VS1 } & VS2 & \multirow{2}{*}{$\mathrm{n}$} & VS3 & \multicolumn{2}{|c|}{ VS4 } & \multirow{2}{*}{$\frac{\mathrm{vtic}}{\left(\mathrm{m}^{3} . \mathrm{cc}\right)}$} \\
\hline$(\mathrm{cm})$ & $(\mathrm{m})$ & $(\mathrm{m})$ & & $\%$ & $\mathrm{n}$ & $\%$ & & $\%$ & $\left(\mathrm{~m}^{3} \cdot \mathrm{cc}\right)$ & $\%$ & \\
\hline \multirow{3}{*}{70,0} & 18,7 & 13,6 & 2 & 86,7 & 0 & 0,0 & 1 & 11,2 & 0,0799 & 2,1 & 3,7430 \\
\hline & 20,7 & 15,6 & 2 & 79,5 & 1 & 12,6 & 0 & 0,0 & 0,3243 & 7,9 & 4,1161 \\
\hline & 22,7 & 17,6 & 2 & 73,7 & 2 & 22,1 & 0 & 0,0 & 0,1865 & 4,2 & 4,4756 \\
\hline \multirow{3}{*}{72,0} & 18,8 & 13,7 & 2 & 86,0 & 1 & 13,5 & 0 & 0,0 & 0,0186 & 0,5 & 4,0018 \\
\hline & 20,8 & 15,7 & 2 & 78,9 & 1 & 12,6 & 0 & 0,0 & 0,3713 & 8,4 & 4,3955 \\
\hline & 22,8 & 17,7 & 3 & 95,4 & 0 & 0,0 & 0 & 0,0 & 0,2217 & 4,6 & 4,7752 \\
\hline \multirow{3}{*}{74,0} & 18,9 & 13,8 & 2 & 85,4 & 1 & 13,5 & 0 & 0,0 & 0,0461 & 1,1 & 4,2696 \\
\hline & 20,9 & 15,8 & 2 & 78,4 & 1 & 12,6 & 1 & 8,9 & 0,0063 & 0,1 & 4,6844 \\
\hline & 22,9 & 17,8 & 3 & 94,9 & 0 & 0,0 & 0 & 0,0 & 0,2592 & 5,1 & 5,0843 \\
\hline \multirow{3}{*}{76,0} & 19,0 & 14,0 & 2 & 84,8 & 1 & 13,6 & 0 & 0,0 & 0,0756 & 1,7 & 4,5464 \\
\hline & 21,0 & 16,0 & 2 & 77,8 & 1 & 12,7 & 1 & 8,9 & 0,0305 & 0,6 & 4,9827 \\
\hline & 23,0 & 18,0 & 3 & 94,5 & 0 & 0,0 & 0 & 0,0 & 0,2989 & 5,5 & 5,4034 \\
\hline \multirow{3}{*}{78,0} & 19,1 & 14,1 & 2 & 84,2 & 1 & 13,6 & 0 & 0,0 & 0,1071 & 2,2 & 4,8322 \\
\hline & 21,1 & 16,1 & 2 & 77,3 & 1 & 12,7 & 1 & 8,9 & 0,0565 & 1,1 & 5,2905 \\
\hline & 23,1 & 18,1 & 3 & 94,1 & 0 & 0,0 & 0 & 0,0 & 0,3409 & 5,9 & 5,7324 \\
\hline \multirow{3}{*}{80,0} & 19,1 & 14,2 & 2 & 83,6 & 1 & 13,6 & 0 & 0,0 & 0,1407 & 2,7 & 5,1271 \\
\hline & 21,1 & 16,2 & 3 & 100,0 & 0 & 0,0 & 0 & 0,0 & 0,0000 & 0,0 & 5,6298 \\
\hline & 23,1 & 18,2 & 3 & 93,7 & 0 & 0,0 & 0 & 0,0 & 0,3851 & 6,3 & 6,0713 \\
\hline
\end{tabular}

Em que: DAP = diâmetro a altura do peito, em $\mathrm{cm} ; \mathrm{h}=$ altura total, em $\mathrm{m}$; hic = altura na inserção da copa, em $\mathrm{m} ; \mathrm{VS}$, VS2, VS3 = proporção do volume cada sortimento em relação ao total compreendido até vtic, em \%; VS4 = volume residual entre a última seção com restante do fuste, $\mathrm{em}^{3}$.cc; vtic = volume total até o ponto de inserção da copa, em $\mathrm{m}^{3}$.cc.

foi restrita à amplitude de diâmetros e alturas observados e empregou o modelo de Kozak (Eq.4). As classes de sortimentos foram estabelecidas arbitrariamente podendo, entretanto, ser alteradas conforme desejado, sem necessidade de reestimativa dos coeficientes. $\mathrm{O}$ volume de cada sortimento foi calculado pelo modelo de Kozak (Eq.4), expresso em valores relativos e em número de toras para os sortimentos S1, S2 e S3 em relação ao volume total do fuste da árvore. O sortimento S4 destinado à energia ou indústria foi expresso em metros cúbicos e também em valores relativos em relação ao volume do fuste (Tabela 7).

Assim, uma árvore de araucária com 20 $\mathrm{cm}$ de diâmetro e 10,4 $\mathrm{m}$ de altura apresentou 0,0\% de volume em sortimentos S1 a S3 e $100 \%$ de sortimento classificado como madeira para energia ou indústria, $\mathrm{S} 4$, com $0,1510 \mathrm{~m}^{3} . \mathrm{cc}$ (Tabela 7). Na mesma relação, uma árvore com diâmetro de 78 $\mathrm{cm}$ e $21,1 \mathrm{~m}$ de altura apresentou duas toras em S1, $77,3 \%$ do volume do fuste, uma tora em S2 com 12,7 $\%$ do volume do fuste, uma tora em S3 com $8,9 \%$ do volume e, em S4 foram classificados $0,0565 \mathrm{~m}^{3} . c c$ ou $1,1 \%$ do volume do fuste compreendido do nível do solo ao ponto de inserção da copa, 5,2905 $\mathrm{m}^{3}$.cc.

\section{CONCLUSÕES}

O modelo da relação altura/DAP e do diâmetro no ponto de inserção da copa obtiveram boas estatísticas de ajuste e precisão, permitindo sua aplicação em atividades destinadas ao planejamento e inventários florestais.

A flexibilidade e a eficiência do modelo de Kozak (1988) em predizer diâmetros e volumes até o ponto de inserção da copa favorecem o uso e a obtenção de sortimentos de madeira de araucária.

\section{REFERÊNCIAS BIBLIOGRÁFICAS}

BIGING, G. S. Taper equations for second mixedconifers of Northean California. Forest Science, Bethesda, v. 30, p. 1103-1117, 1984.

BURKHART, H. E.; TOMÉ, M. Modeling Forest Trees and Stands. Dordrecht: Springer, 2012. $457 \mathrm{p}$.

CLARK, N. A. et al. A review of past research on dendrometers. Forest Science, Bethesda, v. 46, p. 570-576, 2000.

EMBRAPA. Sistema brasileiro de classificação de solos. Brasília: Embrapa Produção de Informação; Rio de Janeiro: Embrapa Solos, 1999. 412 p.

COSTA, E. A.; FINGER, C. A. G.; CUNHA, T. A. Influência da posição sociológica na relação 
hipsométrica de Araucaria angustifolia. Revista Brasileira de Ciências Agrárias, Recife, v. 9, p. 110-116, 2014.

EPAGRI. Dados e Informações Biofísicas da Unidade de Planejamento Regional Planalto Sul Catarinense - UPR 3. Florianópolis: EPAGRI, $2002.70 \mathrm{p}$.

FIGUEIREDO-FILHO, A. et al. Taper equations for Pinus Taeda plantations in Southern Brazil. Forest Ecology and Management, Amsterdam, v. 83, p. 39-46, 1996.

HEINSDIJK, D. Volumes do Pinheiro - tabelas de volume e outros dados sobre o pinheiro brasileiro no Estado de Santa Catarina. Anuário Brasileiro de Economia Florestal, n. 11. Rio de Janeiro: Instituto Nacional do Pinho, 1959. p. 189.

HESS, A. F. et al. Proposta de manejo de Araucaria angustifolia (Bertol.) Kuntze utilizando o quociente de Liocourt e análise de incremento, em propriedade rural no Município de Lages, SC. Pesquisa Florestal Brasileira, Colombo, v. 30, p. 337-345, 2010.

KALLIOVIRTA, J. et al. Evaluation of the Laserrelascope. Forest Ecology and Management, Amsterdam, v. 20, p.181-194, 2005.

KLOS, R. J. et al. Taper equations for five major commercial tree species in Manitoba, Canada. Western Journal of Applied Forestry, Bethesda, v. 22, p. 163-170, 2007.

KOZAK, A. A variable-exponent taper equation. Canadian Journal of Forest Research, Ottawa, v. 18, p. 1363-1368, 1988.

Effects of upper stem measurements on the predictive ability of variable-exponent taper equations. Canadian Journal of Forest Research, Ottawa, v. 28, p. 1078-1083, 1998.

KOZAK, A.; MUNRO, D. D.; SMITH, J. H. G. Taper functions and their application in forest inventory. Forestry Chronicle, Ottawa, v. 45, p. 278-283, 1969.

LEE, W. K. et al. Modeling stem profiles for Pinus densiflora in Korea. Forest Ecology and Management, Amsterdam, v. 172, p. 69-77, 2003. LI, R. et al. Regional stem taper equations for eleven conifer species in the Acadian Region of North America: Development and Assessment. Northern Journal of Applied Forestry, Bethesda, v. 29, p. 5-14, 2012.

LI, R.; WEISKITTEL, A. R. Comparison of model forms for estimating stem taper and volume in the primaryconiferspecies oftheNorthAmericanAcadian Region. Annals Forest Science, Les Ulis, v. 67, p. 302-317, 2010.
MACHADO, S. A. et al. Comportamento da relação hipsométrica de Araucaria angustifolia no capão da Engenharia Florestal da UFPR. Pesquisa Florestal Brasileira, Colombo, n. 56, p. 5-16, 2008.

MAX, T. A., BURKHART, H. E. Segmented polynomial regression applied to taper equations. Forest Science, Bethesda, v. 22, p. 283-288, 1976. MIGUEL, E. P. et al. Modelos polinomiais para representar o perfil e o volume do fuste de Eucalyptus urophylla na região norte do estado de Goiás. Floresta, Curitiba, v. 41, p. 355-368, 2011.

MUHAIRWE, C. K. et al. Effects of adding tree, stand, and site variables to Kozak's variableexponent taper equation. Canadian Journal of Forest Research, Ottawa, v. 24, p. 252-259, 1994.

MÜLLER, I. Forma de fuste e sortimentos de madeira para Eucalyptus grandis hill ex maiden, manejado em alto fuste, na região sudeste do estado do Rio Grande do Sul. 2004. 165 f. (Doutorado em Engenharia Florestal) Universidade Federal de Santa Maria, Santa Maria, 2004.

NIGH, G.; SMITH, W. Effect of climate on lodgepole pine stem taper in British Columbia, Canada. Forestry, Oxford, v. 85, p. 579-587, 2012. PARKEY, R. C.; MARTNEY, T. G. Comparison of Optical Dendrometers for Prediction of Standing Tree Volume. Southern Journal of Applied Forestry, Bethesda, v. 23, p. 407-417, 1998.

SAS INSTITUTE. The SAS System for Microsoft Windows: release 9.1.2. Cary: SAS Institute, 2004. SCHNEIDER, P. R. Betriebswirtschaftliche und ertragskundliche Grundlagen der Forteinnrichtung in Südbrasilien am Beispiel von Pinus elliottii. Diss. d. Albert-Ludwigs Universitat Freiburg, 1984. 190 p. et al. Forma de fuste e sortimentos de madeira de Eucalyptus grandis Maiden para o estado do Rio Grande do Sul. Ciência Florestal, Santa Maria, v. 6, p. 79-88, 1996.

SCHNEIDER, P. S. P. Estrutura bioeconômica da produção no manejo da densidade de Pinus taeda L. na região do Planalto Catarinense, Brasil. 2012. 188 f. (Doutorado em Engenharia Florestal) Universidade Federal de Santa Maria, Santa Maria, 2012.

SHARMA, M.; PARTON, J. Modeling stand density effects on taper for jack pine and black spruce plantations using dimensional analysis. Forest Science, Bethesda, v. 55, p. 268-282, 2009.

SHARMA, M.; ZHANG, S. Y. Variable-exponent taper equations for jack pine, black spruce, and 
balsam fir in eastern Canada. Forest Ecology and Management, Amsterdam, v. 198, p. 39-53, 2004. SOUZA, C. A. M. Modelos de afilamento para Pinus taeda L. ajustados segundo a forma do fuste e métodos de estratificação. 2009. 120 f. Tese (Doutorado em Engenharia Florestal) Universidade Federal de Santa Maria, Santa Maria, 2009.

et al. Eficiência de um modelo de afilamento ajustado sem e com estratificação por classe de quociente de forma para formação de sortimentos de Pinus taeda L. Ciência Florestal, Santa Maria, v. 22, p. 125-135, 2012.

THOMAS, G. B.; WEIR, M. D.; HASS, J. Thomas' Calculus: Early Transcendentals. 12. ed. [s. 1.]: Addison Wesley, 2010. 1211 p.

WILLIAMS, M. S. et al. Evaluation of the Barr \& Stroud FP15 and Criterion 400 Laser Dendrometers for Measuring Upper Stem Diameters and Heights. Forest Science, Bethesda, v. 45, p. 53-61, 1999. 